\title{
Representação social sobre o aprendizado de estudantes de Odontologia da Universidade Federal do Maranhão inseridos em Estágio Curricular na Atenção Primária à Saúde
}

\author{
Francenilde Silva de Sousa*; Aline Sampieri Tonello**; Judith Rafaelle Oliveira Pinho***
}

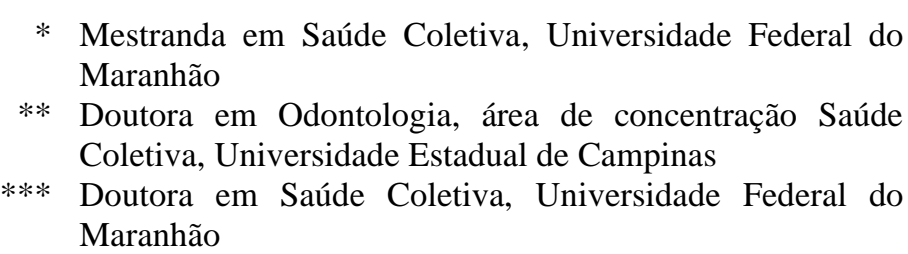

Recebido: 03/04/2020. Aprovado: 25/01/2021.

\begin{abstract}
RESUMO
O objetivo deste estudo foi identificar a representação social sobre o aprendizado de estudantes do curso de Odontologia da Universidade Federal do Maranhão, ao final de um estágio curricular no contexto da Atenção Primária à Saúde, e comparar os resultados com as Diretrizes Curriculares Nacionais vigentes. Foi realizado um estudo de natureza qualitativa, por meio de um questionário, instrumento utilizado para avaliar o estágio, do qual somente a primeira questão era de interesse para este estudo. Nessa questão era solicitado ao aluno que evocasse, em ordem crescente de importância, cinco palavras que demonstrassem seu aprendizado ao fim do estágio. O questionário foi preenchido pelos egressos do $5^{\circ}$ período entre 2016 e 2019, totalizando 100 avaliações. Foi considerada uma abordagem estrutural e Análise Prototípica, na qual se considera a frequência e a ordem das palavras, compondo um núcleo central e periferias. O software utilizado, o OpenEVOC®, identificou 500 termos evocados e 10 que formaram a representação social. $\mathrm{O}$ núcleo central foi composto pelos termos: planejamento, conhecimento e gestão; a primeira periferia: organização; na zona de contraste: territorialização e humanização; e segunda periferia: comprometimento, realidade, paciência e metas. As evocações sugerem que o estágio foi composto por atividades que dotassem os profissionais de competências e habilidades necessárias do novo perfil de profissional. A representação social dos estudantes do curso de Odontologia, inseridos em um estágio ambientado na Atenção Primária à Saúde, é marcada por termos característicos da implementação de conhecimentos do sistema público de saúde brasileiro e reflete conteúdos conexos com as diretrizes curriculares do curso.
\end{abstract}

Descritores: Educação em Saúde. Currículo. Associação Livre. 


\section{INTRODUÇÃO}

Há forte tendência para que haja renovação nos currículos das ciências da saúde para acompanhar as necessidades sociais emergentes, práticas educacionais e evitar estagnação ${ }^{1}$. No Brasil, na região sul, existem estudos que avaliaram, utilizando técnicas qualitativas ou quantitativas, a percepção de estudantes frente a essas mudanças nos projetos pedagógicos do curso de Odontologia e foi evidenciada grande valorização de estágios no Sistema Único de Saúde $(\mathrm{SUS})^{2,3}$.

Em 2002 foram implantadas as primeiras Diretrizes Curriculares Nacionais (DCN) para cursos de graduação em Odontologia pelo Ministério da Educação ${ }^{4}$, sendo revisada em $2019^{5}$, de acordo com a Lei de Diretrizes e Bases da Educação Nacional (LDB) ${ }^{6}$. Segundo as DCN, o perfil do cirurgião-dentista deve ser de um profissional generalista, com princípios que o preparam para atuação refletida na realidade social do ambiente inserido e contemplem os determinantes sociais da saúde (DSS) com foco no SUS $^{4,7}$.

Visando a um caminho como resposta a esse desafio proposto, os cursos de Odontologia do país buscaram se adequar ao novo perfil por meio de mudanças nos currículos, ajustando conteúdo a ser ministrado e buscando avaliar as alterações $\operatorname{propostas}^{2,7,8}$. Nesse sentido, o curso de Odontologia da Universidade Federal do Maranhão (UFMA), em 2011, implantou um novo projeto pedagógico propondo a inserção dos estudantes em práticas integradas de ensinoserviço-comunidade 9 .

A inquietação, no entanto, é saber se tais mudanças trouxeram avanço efetivo na realidade do ensino, o que significa ir além da reorganização de conteúdos, disciplinas, cargas horárias e tempo de duração dos cursos. É necessário repensar o verdadeiro sentido dos cursos de Odontologia dentro do projeto pedagógico, que é influenciado pelas $\mathrm{DCN}^{10}$, e em como avaliar as mudanças sob diferentes olhares, como o olhar dos estudantes ${ }^{11}$.

Ao avaliar uma das ferramentas de mudanças curriculares, o estágio curricular no contexto da Atenção Primária à Saúde (APS), este estudo pretende contribuir com evidências para $\mathrm{o}$ aprendizado no cenário da prática e subsidiar tomadas de decisão na gestão pedagógica. É importante que a avaliação seja correspondente ao conteúdo, que foi prático e, assim, a representação social, considerada uma forma de pensar ligada à ação para Moscovici (1988) $)^{12}$, permite compreender potencialidades e limitações no cenário a ser avaliado. A Teoria das Representações Sociais $(\mathrm{TRS})^{13}$ tem sido empregada de forma ampla em estudos na área da saúde ${ }^{14-17}$. Possui grande diversidade de métodos e técnicas de análise ${ }^{13}$ e uma delas é a da Análise Prototípica, na qual se considera a frequência e a ordem de evocação de palavras ${ }^{18}$.

Exposto esse cenário, o objetivo deste trabalho foi identificar a representação social sobre o aprendizado de estudantes do curso de Odontologia da Universidade Federal do Maranhão, ao final de um estágio curricular contextualizado na APS, e comparar os resultados com as DCN vigentes.

\section{METODOLOGIA}

\section{Equipe de pesquisa e reflexividade}

A coleta das evocações foi realizada a partir de um instrumento de avaliação do estágio aplicado pelas docentes ao final do semestre letivo. Não havia a possibilidade de identificação do estudante.

\section{Desenho do estudo}

Foi realizado um estudo de natureza qualitativa, com base nas representações sociais e abordagem estrutural. Minayo (2012) ${ }^{19}$ demonstra que a pesquisa qualitativa possibilita 
a exploração da realidade mediante o aprofundamento de significados, atitudes, crenças, valores e relações humanas, que não seria possível por meio de um estudo unicamente quantitativo. Moscovici $(1988)^{12}$ entende a representação social como modo de pensar ligado à ação, no qual se criam categorias cognitivas e relações de sentido do que se é vivido. A abordagem estrutural pressupõe a existência de uma estrutura nas representações, elaborada por grupos sociais e estruturada por um núcleo central, resistente a mudanças; periferias próximas ao núcleo, que o protegem quanto a mudanças; e periferia mais distante que revela o diferente ou não compartilhado pela maioria $^{20}$.

A população consistiu em todos os egressos do estágio curricular supervisionado (ECS) II, do $5^{\circ}$ período, durante os semestres compreendidos de 2016 a 2019. Foram incluídos todos os estudantes que cursaram até o fim do estágio e foram excluídos todos os que trancaram o curso ou não chegaram ao fim. Houve um total de 100 (cem) estudantes do curso de Odontologia da UFMA. Não houve perdas por falta, devido estágio ser totalmente presencial; nem por recusa dos alunos, pois todos compreenderam seu importante papel no desenvolvimento da avaliação do estágio.

De acordo com o projeto pedagógico da UFMA $^{9}$, o estágio curricular supervisionado II possui a carga horária total de 45 horas, sendo 3 horas semanais. É ofertado ao Departamento de Odontologia pelo Departamento de Saúde Pública e é ambientado na APS. Composto geralmente por 4 (quatro) professoras doutoras em Saúde Coletiva e média de 15 (quinze) alunos por semestre.

Possui o objetivo de proporcionar ao acadêmico de Odontologia uma vivência no SUS, integrando-o à Equipe de Saúde na Família (ESF), capacitando-o para solucionar problemas frequentes surgidos na prática profissional em serviços públicos. Além da sensibilização para o trabalho de educação em Saúde Coletiva e integrar a Odontologia a uma equipe multiprofissional de assistência à saúde da população. Assim, são realizadas visitas a Unidades Básicas de Saúde (UBS), a escolas próximas a elas e ligadas pelo Programa Saúde na Escola (PSE), a órgãos de avaliação em saúde como a vigilância sanitária, além de aulas com metodologias ativas com uso de Problem Based Learning (PBL) e de práticas em laboratórios de informática. Assim, é centralizado na ambientação da APS.

As atividades desenvolvidas estão relacionadas com o descrito na ementa, cuja idealização é dotar o aluno de habilidades e competências em diagnóstico em saúde, sistema de avaliação dos serviços, noções de planejamento e organização dos serviços, conceitos teóricos sobre territorialização, planejamento de levantamento epidemiológico em saúde bucal, protocolo de pesquisa, construção de instrumento de coleta e de banco de dados, recursos humanos em saúde bucal. A avaliação é feita por meio da frequência, participação e desenvoltura dos alunos nas atividades desenvolvidas.

Toda a caracterização das etapas do ECS II está de acordo com o que foi descrito por Pinho e colaboradores (2019) ${ }^{21}$, professores e alunos que colaboraram na elaboração de uma atividade desenvolvida durante estágio. Da implantação até a execução houve as seguintes etapas: concepção pedagógica, articulação político/ administrativa, planejamento estratégico, monitoramento e avaliação.

A concepção pedagógica refere-se ao planejamento do estágio com objetivo de executar intervenções a partir de problemas identificados no território no âmbito da Atenção Básica, articulando com os DSS. 
A articulação político/administrativa consiste no contato com as instâncias de gestão para inserção dos alunos no SUS. Para tal foram realizadas reuniões com a Secretaria Municipal de Saúde (SEMUS), das quais resultaram nos produtos: autorização formal para realização das atividades, pactuação de fluxos de informação e periodicidade de devolutiva de resultados e/ou achados.

O planejamento estratégico inclui todas as etapas desse processo, focando na elaboração das atividades. A etapa relativa ao monitoramento e avaliação do estágio corresponde ao acompanhamento pedagógico do aluno e de suas percepções sobre seu aprendizado. Desse modo, foi instituído um instrumento de avaliação aplicado no último dia do estágio, com objetivo de identificar dificuldades vividas e propor melhorias pedagógicas no seu planejamento.

Os dados deste estudo foram coletados por meio de um questionário, instrumento utilizado para avaliar o estágio e composto por 8 (oito) perguntas. A primeira questão, a de interesse para este estudo, pedia ao aluno que ele evocasse 5 (cinco) palavras, em ordem crescente de importância, que expressassem seu aprendizado após o estágio curricular II.

Essas palavras são as evocações, utilizadas neste estudo, escritas após o disparador (o enunciado da questão) ${ }^{20}$. Todas as demais pediam ao aluno para selecionar a opção mais pertinente para ele, dentre concordo fortemente, concordo, indiferente, discordo ou discordo fortemente, sobre o uso do PBL.

Não foi pedida assinatura em Termo de Consentimento Livre e Esclarecido para garantir o anonimato e liberdade de avaliação, sem receio de retaliações. Foi aplicado no último dia do estágio, permitindo toda a experiência ser avaliada, e dado um tempo de 5 (cinco) minutos, pois a primeira questão não buscava respostas bem elaboradas e sim a espontaneidade ${ }^{20}$.

\section{Análise e descoberta}

As respostas da primeira questão foram analisadas por meio da abordagem estrutural e pela Análise Prototípica, na qual resulta no núcleo central e sistemas periféricos. Ambos sistemas são compostos pela frequência e ordem das evocações no corpus de representação social $^{20}$.

Os dados foram digitados em planilha e analisados pelo software OpenEVOC®, seguindo etapas de acordo com Wachelke e Wolter $(2011)^{18}$. Toda a análise foi feita após o fim das atividades da turma do primeiro semestre de 2019.

A primeira etapa, que ocorreu durante digitação da planilha, foi a da lematização. Ela consiste em padronizar palavras que compartilhem o mesmo radical e que na prática possuem o mesmo sentido (ex.: humanizado e humanização = humanização).

A segunda foi a seleção dos pontos de corte: o da maior frequência foi pré-determinado considerando a maior comum das zonas de alta frequência, 5\%; o da ordem de evocação, haja vista ser um número ímpar (5), foi considerado sua mediana, 3 (três); e a frequência mínima para inclusão nos quadrantes, $2 \%$.

A terceira, detalhada na figura 1 , foi a descrição e interpretação dos dados obtidos, considerando núcleo central e sistemas periféricos. A análise foi realizada à luz do confronto entre o resultado, desencadeado pelas palavras evocadas que se referiam ao ECS II, e as DCN do curso de Odontologia.

\section{Considerações éticas}

O desenvolvimento desta pesquisa seguiu os requisitos da Resolução 466/12 do Conselho Nacional de Saúde/Ministério da Saúde do Brasil e suas complementares, que regulamentam as pesquisas envolvendo seres humanos, e obteve aprovação do Comitê de Ética em Pesquisa da UFMA com o parecer $n^{\circ}$ 3.658.417. 


\begin{tabular}{|l|l|}
\hline $\begin{array}{l}\text { Núcleo central (+ +): onde se encontram as evocações de } \\
\text { maior frequência e ordem de evocação inferior à média } \\
\text { geral das evocações, e que correspondem aos elementos } \\
\text { de maior probabilidade de pertencer ao núcleo central. }\end{array}$ & $\begin{array}{l}\text { Sistema periférico (+ -): evocações de maior frequência e } \\
\text { maior ordem de evocação, começa como uma primeira } \\
\text { coroa do sistema periférico onde se encontram evocações } \\
\text { muito citadas, mas sem importância para os sujeitos. }\end{array}$ \\
\hline $\begin{array}{l}\text { Sistema periférico (- +): evocações de menor frequência e } \\
\text { de menor ordem de evocação, somando uma segunda }\end{array}$ & $\begin{array}{l}\text { Sistema periférico (- -): evocações de menor frequência e } \\
\text { maior ordem de evocação, consideradas a última coroa } \\
\text { coroa do sistema periférico onde se encontram as as } \\
\text { evocações consideradas importantes por um pequeno } \\
\text { grupo de sujeitos. }\end{array}$ \\
irrelevantes para a representação.
\end{tabular}

Figura 1. Distribuição dos resultados da análise prototípica. Fonte: Pereira (2001) ${ }^{12}$

\section{RESULTADOS}

Dos 100 questionários respondidos, o software OpenEVOC $®$ identificou 500 (quinhentos) termos evocados da questão em interesse, sendo 129 (cento e vinte e nove) distintos. Após aplicação dos pontos de corte, foram 10 (dez) evocações responsáveis por formar núcleo central e sistemas periféricos.
A análise combinada da frequência e ordem das evocações possibilitou a construção e identificação do núcleo central e sistemas periféricos que dão sentido à representação social do aprendizado adquirido pelos estudantes do curso de Odontologia após o estágio supervisionado no contexto da APS (tabela 1).

Tabela 1. Representação social do aprendizado dos estudantes do curso de Odontologia após estágio supervisionado no contexto da Atenção Primária à Saúde

\begin{tabular}{|c|c|c|c|c|c|}
\hline \multicolumn{3}{|c|}{ (++) Núcleo Central } & \multicolumn{3}{|c|}{ (+-) Sistema Periférico (primeira periferia) } \\
\hline $\begin{array}{l}\text { Frequência } \\
\qquad \%) \geq 5\end{array}$ & $\begin{array}{c}\text { Termo } \\
\text { evocado }\end{array}$ & $\begin{array}{c}\text { Ordem de } \\
\text { evocação < } 3\end{array}$ & $\begin{array}{l}\text { Frequência } \\
\quad(\%) \geq 5\end{array}$ & $\begin{array}{c}\text { Termo } \\
\text { evocado }\end{array}$ & $\begin{array}{c}\text { Ordem de } \\
\text { evocação } \geq 3\end{array}$ \\
\hline 11,2 & Planejamento & 2,04 & & & \\
\hline 5,6 & Conhecimento & 2,96 & 5 & Organização & 3,08 \\
\hline 5,4 & Gestão & 2,74 & & & \\
\hline \multicolumn{3}{|c|}{ (-+) Sistema Periférico (zona de contraste) } & \multicolumn{3}{|c|}{ (--) Sistema Periférico (segunda periferia) } \\
\hline $\begin{array}{l}\text { Frequência } \\
(\%)<5\end{array}$ & $\begin{array}{c}\text { Termo } \\
\text { evocado }\end{array}$ & $\begin{array}{c}\text { Ordem de } \\
\text { evocação }<3\end{array}$ & $\begin{array}{l}\text { Frequência } \\
\qquad(\%)<5\end{array}$ & $\begin{array}{c}\text { Termo } \\
\text { evocado }\end{array}$ & $\begin{array}{c}\text { Ordem de } \\
\text { evocação } \geq 3\end{array}$ \\
\hline \multirow{2}{*}{3,2} & \multirow{2}{*}{ Territorialização } & \multirow{2}{*}{2,38} & 3 & Comprometimento & 3 \\
\hline & & & 2,2 & Realidade & 3,45 \\
\hline \multirow{2}{*}{2,4} & \multirow{2}{*}{ Humanização } & \multirow{2}{*}{2,33} & 2,2 & Paciência & 3,64 \\
\hline & & & 2 & Metas & 4,1 \\
\hline
\end{tabular}

$\mathrm{Na}$ formação do núcleo central estão os termos evocados (planejamento, conhecimento e gestão) com maior frequência e em menor ordem de evocação, sendo os que apresentam uma base comum aos estudantes. Na primeira periferia (organização), os que possuem maior frequência, contudo foram mais evocados entre os últimos termos, podendo ser termos considerados irrelevantes quando comparados com os do núcleo central (tabela 1).

Na zona de contraste há os temos evocados (territorialização e humanização) com menores frequência e ordem de evocação e leva essa nomeação por ser oposto ao núcleo central, 
possuindo relevância apenas para um pequeno grupo dentro dos estudantes. Já na segunda periferia os termos de menor frequência e maior ordem de evocação (comprometimento, realidade, paciência e metas), que também são consideradas sem grande relevância para a representação, contudo necessários para confrontar com núcleo central (tabela 1).

\section{DISCUSSÃO}

As evocações que representaram prontamente o aprendizado dos alunos ao final do estágio foram: planejamento, conhecimento e gestão. Estas e as demais, das periferias, estão consoante aos objetivos das DCN do curso de Odontologia de dotar o cirurgião-dentista de conhecimentos que cercam a atenção à saúde, tomada de decisões, comunicação, liderança, gestão em saúde e educação permanente ${ }^{5}$.

O termo "planejamento" é conceituado como processo de racionalização, visando solucionar problemas e atender necessidades individuais e coletivas ${ }^{22}$. Conforme o descrito nas DCN como uma referência de conteúdo a ser trabalhada na teoria e prática, além de estar inserido em uma das competências de um profissional egresso, a de gestão à saúde ${ }^{4}$.

O termo "conhecimento" pode ser apreendido como termo estimulante que amplia o espaço para pluralidade nos momentos de resolver problemas ${ }^{23}$, como na ESF, cuja atenção à saúde deve ser multiprofissional e, apesar de alguns entraves, a saúde bucal foi integrada a ela ${ }^{11,24,25}$ e também é reflexo de uma das competências das DCN - educação permanente na qual o conhecimento deve ser renovado continuamente ${ }^{4}$.

O termo "gestão" pode se enquadrar em resultados positivos através de melhorias na articulação de inúmeras variáveis nas redes de saúde, incluindo a bucal ${ }^{26,27}$, e reforça que o cirurgião-dentista generalista deve apresentar competência de gerenciar e administrar recursos físicos e humanos com capacidade para se tornar gestor $^{4}$. Isso se opõe ao modelo de formação anterior no qual era centrado nas ciências técnicas e evidencia a nova formação, corroborando resultados esperados com o novo modelo ${ }^{8,28,29}$.

Essas evocações sugerem que o planejamento das atividades do estágio em questão foi baseado considerando novo perfil do cirurgião-dentista. Pois, ainda que os termos possuam amplos conceitos e entendimentos, todos estão previstos no projeto pedagógico e este foi elaborado fundamentado nas DCN.

Os sistemas periféricos possuem as funções de concretização, regulação e defesa, permitindo apresentar materialização, mobilidade e evolução da representação social ${ }^{30}$. Na prática representa um complemento ao núcleo central, auxiliando na sua compreensão.

$\mathrm{Na}$ primeira periferia, a palavra evocada (organização) não é considerada com tanta relevância, pois ainda que evocada com mesma frequência do núcleo central, foi relembrada entre as derradeiras ${ }^{31}$. Pode ser entendido como um termo enraizado entre os estudantes na tentativa de acompanhar as alterações e torná-lo mais apropriado ${ }^{32}$.

Sugere-se que ao participar de atividades juntamente com a equipe do PSE, os estudantes possam ter desenvolvido e praticado a organização durante o planejamento dessas atividades. Os estudantes de odontologia deveriam durante o estágio, realizar o diagnóstico situacional afim de conhecer o território para poder planejar e realizar ações de saúde bucal para aquela população.

A zona de contraste possui essa nomenclatura por se contrastar com a frequência do núcleo central ${ }^{31}$. Os termos também são apresentados em menor ordem de evocação, porém com menor frequência, caracterizando um 
grupo de palavras que se sobressaíram para um pequeno grupo dos estudantes. São evocações (territorialização e humanização) condizentes com o que se espera dos novos egressos do curso, para que possuam forte relação com o sistema público de saúde, ampliando os campos de prática no SUS com potencial para qualificar distintas extensões do perfil profissional a ser formado no país ${ }^{4}$.

O termo "territorialização" é reflexo de uma ferramenta de transição do modelo tecnicista e biologista para reorientação dentro do sistema público de saúde de modo fluido e funcional $^{33}$. Sua importância está relacionada com a identificação de aspectos ambientais, demográficos, econômicos, espirituais e sociais e dos problemas mais evidentes em determinada área para que sejam identificados e levados em conta os DSS ${ }^{34}$.

A humanização foi uma palavra evocada que possui significado e aplicação fundamentais para o processo de produção de saúde ${ }^{35}$ por representar amenização na realidade, criação de vínculos harmoniosos e respeitosos e de leveza no atendimento como devolutiva para os usuários $^{27}$. As duas evocações da zona de contraste se relacionam diretamente com o contexto da Estratégia Saúde da Família, visto que o processo de trabalho das eSF envolve definir o território de atuação e população sob responsabilidade das UBS e das equipes (territorialização); além de realizar o acolhimento com escuta qualificada, classificação de risco, avaliação de necessidade de saúde e análise de vulnerabilidade (humanização) $)^{36}$.

Na segunda periferia há palavras evocadas de menor frequência e maior ordem, sendo, assim, as mais irrelevantes por terem sido pouco recordadas e quando foram, estavam em colocações derradeiras. Contudo são termos (comprometimento, realidade, paciência e metas) complementares ao que se anseia para nova formação e proximidade do SUS $^{37-39}$, demonstram que foram desenvolvidas em algum momento durante o período do estágio e foram marcantes de forma mais individual ${ }^{20}$.

Ademais, a representação social do aprendizado de estudantes de Odontologia da UFMA faz um paralelo com grande parte das atribuições de cirurgiões-dentistas que atuam na APS. Dentre elas as de: i) realizar diagnóstico com finalidade de obter perfil epidemiológico para o planejamento e a programação em saúde bucal; ii) coordenar e participar de ações coletivas voltadas à promoção da saúde e à prevenção de doenças bucais; iii) acompanhar, apoiar e desenvolver atividades referentes à saúde bucal com os demais membros da equipe, buscando aproximar e integrar ações de saúde de forma multidisciplinar; iv) realizar supervisão técnica do auxiliar e do técnico em saúde bucal; e v) participar do gerenciamento dos insumos necessários para o adequado funcionamento da $\mathrm{UBS}^{36}$.

Embora estudos ainda apontem fragilidades nos projetos pedagógicos dos cursos de Odontologia como, por exemplo, a notoriedade das disciplinas clínicas em detrimento às práticas no SUS, seja por maior carga horária ou mais organização, e a carga horária limitada disponibilizada para os estágios $^{2,40}$, este estudo demonstrou que é possível que tais inquietações sejam minimizadas por meio da elaboração de um projeto pedagógico consoante as DCN. Os termos evocados pelos estudantes, frente a realidade após um estágio no sistema público de saúde, foram favoráveis e corroboram com um aprendizado ampliado.

Há necessidade de se avançar na consolidação do currículo integrado ${ }^{3,29}$ e na utilização de metodologias ativas no processo pedagógico ${ }^{41,42}$, desde a elaboração até avaliação 
das atividades. Embora não sejam encontrados com facilidade estudos com essa metodologia na área da Odontologia, o uso da análise qualitativa contribui para discussões e suscita novas investigações ${ }^{29,31}$ acerca das representações sociais no campo da formação na saúde bucal.

Ademais, os aspectos positivos deste estudo revelam diferentes olhares a partir da percepção de situações reais e experiências passadas por cada aluno; proporcionam a avaliação do estágio de uma perspectiva distinta, levando em consideração a formação de um cirurgião-dentista generalista; a corroboração do previsto no planejamento para ser desenvolvido; e enaltecimento do aprendizado dos estudantes.

Aponta-se a necessidade de ampliação de pesquisas nesse sentido para avaliação além dos ECS. Ainda são poucos estudos com delineamento metodológico similar avaliando objeto de estudo análogo, dificultando discussão mais extrovertida, considerando as semelhanças e até mesmo as diferenças das pesquisas. $\mathrm{O}$ que pode ser considerado como limite para o estudo, haja vista ter sido discutido à luz das DCN, contudo, poderia ser enriquecido pelo confronto de outras experiências.

\section{CONCLUSÕES}

A representação social sobre aprendizado de estudantes do curso de Odontologia da Universidade Federal do Maranhão, após a conclusão de estágio no âmbito do SUS, é marcada por termos característicos da implementação de conhecimentos da APS do sistema público de saúde brasileiro. Pelo olhar dos próprios estudantes, o aprendizado do estágio revelou evocações que refletem coerência com o proposto nas DCN vigentes para uma formação generalista e com foco no SUS.

\section{ABSTRACT}

Social representation on the learning of dentistry students from the Federal University of Maranhão included in the curricular internship in Primary Health Care

This study aimed to identify the social representation on the learning of students of the Dentistry course at the Federal University of Maranhão, at the end of a curricular internship in Primary Health Care, and compare the results with the current National Curricular Guidelines. A qualitative study was carried out using a questionnaire, an instrument used to assess the internship, and only the first question was of interest to this study. In this question, students were asked to evoke five words that demonstrated their learning at the end of the internship in increasing order of importance. The questionnaire was completed by graduates from the $5^{\text {th }}$ period between 2016 and 2019, totaling 100 evaluations. A structural approach and Prototypical Analysis were adopted, considering the frequency and order of words, composing a central and peripheral core. Software OpenEVOC® identified 500 evoked terms and 10 that established a social representation. The central core consisted of the following terms: planning, knowledge, and management; the first periphery: organization; the contrast zone: territorialization and humanization; and the second periphery: commitment, reality, patience, and goals. Evocations suggested that the internship consisted of activities that equipped the professionals with the necessary skills and abilities for the new professional profile. The social representation of students in the Dentistry course included in an environmental internship in Primary Health Care is marked by terms typical of implementing knowledge in the Brazilian public health system and shows the course's curricular programs' contents.

Descriptors: Health Education. Curriculum. Free Association.

\section{REFERÊNCIAS}

1. Mcleod PJ, Steinert Y. Twelve tips for curriculum renewal. Med Teach. 2014; 37(3):232-8.

2. Fadel CB, Baldani MH. Percepções de formandos do curso de odontologia sobre 
as diretrizes curriculares nacionais. Trab Educ e Saúde. 2013;11(2):339-54.

3. Silveira JLGC da, Garcia VL. Mudança curricular em odontologia: significados a partir dos sujeitos da aprendizagem. Interface Comunic Saúde Educ. 2015;19(52):145-58.

4. Brasil. Ministério da Educação. Conselho Nacional de Educação. Câmara de Educação Superior. Resolução CNE/CES, de 19 de fevereiro e 2002 [Institui Diretrizes Curriculares Nacionais do Curso de Graduação em Odontologia]. Diário Oficial da União. 2002, 04 de mar; Seção 1:10.

5. Brasil. Ministério da Educação. Conselho Nacional de Educação. Câmara de Educação Superior. Parecer CNE/CES 803/2018. [Diretrizes Curriculares Nacionais do Curso de Graduação em Odontologia]. Diário Oficial da União. 2019, 04 de abr; Seção 1:20.

6. Brasil. Lei de Diretrizes e Bases da Educação Nacional. Lei $n^{0}$ 9394, 20 de dezembro de 1996. In: Diário Oficial da União 1996.

7. Sponchiado Júnior EC, Conde $\mathrm{NC}$ de $\mathrm{O}$, Martins IEB, Carneiro FC, Vieira JMR, Rebelo MAB. Os caminhos da reformulação do Projeto Pedagógico da Faculdade de Odontologia da Universidade Federal do Amazonas. Rev ABENO. 2019;19(2):13-21.

8. Toassi RFC, Stobäus CD, Mosquera JJM, Moysés SJ. Curriculo integrado no Ensino de Odontologia: novos sentidos para a formação na área da saúde. Interface Comunic Saúde Educ. 2012;16(41):529542.

9. Universidade Federal do Maranhão. Projeto pedagógico do curso de Odontologia da Universidade Federal do
Maranhão. Vol. 1. 2011. 108 p.

10. Lemos CLS, Fonseca SG de. Knowledge and curricular practices: an analysis on a university-level healthcare course. Interface Comunic Saúde Educ. 2009;13(28):57-69.

11. Tenório F, Barbosa L, Teixeira SR, Nunes MDF. Implantação das Diretrizes Curriculares Nacionais nos cursos de Odontologia: opinião de formandos de uma universidade pública. Rev da ABENO. 2016;16(4):61-71.

12. Moscovici S. Notes towards a description of Social Representations. Eur J Soc Psychol. 1988;18(3):211-50.

13. Pereira C. Análise de dados qualitativos aplicados às representações sociais: um modelo para estudar as representações sociais. Psicologia. 2001;15(1):177-204.

14. Gomes AMT, Oliveira DC de, Sá CP de. As representações sociais do sistema único de saúde no município do Rio de Janeiro, Brasil, segundo a abordagem estrutural. Rev Lat Am Enfermagem. 2008;16(1):122-9.

15. Moura LM de, Shimizu HE. Representações sociais de conselheiros de saúde acerca do direito à saúde e da cidadania. Rev Gaúcha Enferm. 2017;37(esp):1-9.

16. Ferreira MCG, Tura LFR, Silva RC da, Ferreira M de A. Representações sociais de idosos sobre qualidade de vida. Rev Bras Enferm. 2017;70(4):806-13.

17. Moura SG de, Filha M de OF, Moreira MASP, Simpson CA, Tura LFR, Silva AO. Representações sociais sobre terapia comunitária integrativa construídas por idosos. Rev Gaúcha Enferm. 2017;38(2):1-6.

18. Wachelke J, Wolter R. Critérios de construção e relato da Análise Prototípica 
para Representações Sociais. Psicol Teor e Pesqui. 2011;27(4):521-6.

19. Minayo $\mathrm{MC}$ de $\mathrm{S}$. Análise qualitativa: Teoria, passos e fidedignidade. Ciênc Saúde Coletiva. 2012;17(3):621-6.

20. Mororó LP, Couto MES, Assis RAM de, Bertoni LM, Galinkin AL. Teoria e métodos em Representações Sociais. In: EDITUS, editor. Notas teóricometodológicas de pesquisas em educação: concepções e trajetórias. Ilhéus, BA; 2017. p. 101-22.

21. Pinho JRO, Tonello AS, Thomaz EBAF, Queiroz RC de S, Aguiar BD de, Mendes RJS. Programa Saúde na Escola como campo de práticas para a formação do dentista. In: SUS e Saúde Bucal no Brasil: por um futuro com motivos para sorrir. $1^{\mathrm{a}}$. São Paulo; 2019. p. 96-101.

22. Teixeira CF. Planejamento em Saúde: conceitos, métodos e experiências. EDUFBA, editor. Salvador; 2010. 161 p.

23. Ferreira VN, Pereira IDF. O desafio do conhecimento: pesquisa qualitativa em saúde. J Manag Prim Health Care. 2014;5(2):258-61.

24. Bruder MV, Lolli LF, Palácios AR, Rocha NB, Veltrini VC, Gasparetto A, et al. Estágio supervisionado na odontologia: vivência da promoção da saúde e integração multiprofissional. Rev Bras Promoç Saúde. 2017;30(2):294-300.

25. Toassi RFC, Ely LI. Integração entre currículos na educação de profissionais da saúde: a potência para educação interprofissional na graduação. Interface Comunic Saúde Educ. 2018;22(supl. 2):1563-1575.

26. Brasil. A Saúde Bucal no Sistema Único de Saúde. Brasília: Ministério da Saúde; 2018. 354 p.

27. Alves DF de C, Silva SPC e. Gestão e
Humanização do Serviço de Odontologia na Unidade de Saúde. Id Line Multidiscip Psycology J. 2017;11(36):1-12.

28. Tompsen NN, Meireles E, Peduzzi M, Toassi RFC. Educação interprofissional na graduação em Odontologia: experiências curriculares e disponibilidade de estudantes. Rev Odontol da UNESP. 2018;47(5):309-20.

29. Toassi RFC, Souza JM de, Baumgarten A, Rösing CK. Avaliação curricular na educação superior em odontologia: discutindo as mudanças curriculares na formação em saúde no Brasil. Rev ABENO. 2018;12(2):170-7.

30. Shimizu HE, Silva JR e, Moura LM de, Bermúdez XPD, Odeh MM. A estrutura das representações sociais sobre saúde e doença entre membros de movimentos sociais. Ciênc Saude Colet. 2015;20(9):2899-910.

31. Pereira C. Análise de dados qualitativos aplicados às representações sociais. Psicologia. 2014;15(1):177-204.

32. Azevedo KR, Cerqueira TCS. Jovens com deficiência intelectual nas representações sociais de professores de ensino médio. Psicol Saber Soc. 2015;4(1):34-51.

33. Justo LG, Severo AK de S, Félix-Silva AV, Soares LS, e Silva-Júnior FL, Pedrosa JI dos S. A territorialização na Atenção Básica: um relato de experiência na formação médica. Interface Comunic Saúde Educ. 2017;21(c):1345-54.

34. Araújo GB, Filho FWPA, Santos R da S, Lira RCM. Territorialização em saúde como instrumento de formação para estudantes de Medicina: relato de experiência. SANARE - Rev Políticas Públicas. 2017;16(1):124-9.

35. Carneiro Neto JN, Cordeiro TMSC, Falcão MML. Humanização em saúde e a 
odontologia. Brazilian J Heal Res. 2015;16(2):130-8.

36. Brasil. Portaria $\mathrm{n}^{\mathrm{o}} 2.436$, de 21 de setembro de 2017. Aprova a Política Nacional de Atenção Básica, estabelecendo a revisão de diretrizes para a organização da Atenção Básica, no âmbito do Sistema Único de Saúde (SUS). Brasília; 2017 p. 35.

37. Mattos GCM, Ferreira EF, Leite ICG, Greco RM. A inclusão da equipe de saúde bucal na Estratégia Saúde da família: entraves, avanços e desafios. Ciênc Saude Colet. 2014;19(2):373-82.

38. Finkler M. Formação Ética na Graduação em Odontologia: realidade e desafios. Universidade Federal de Santa Catarina; 2009.

39. Finkler M, Caetano JC, Ramos FRS. Integração "ensino-serviço" na formação profissional em Odontologia. Interface Comunic Saúde Educ. 2011;15(39):1053-
67.

40. Brasil. Lei $\mathrm{n}^{\mathrm{o}} 11.788$, de 25 de setembro de 2008. Diário Oficial da União 1996. 2008. 5 p.

41. Colares KTP, Oliveira W De. Metodologias Ativas na formação profissional em saúde: uma revisão. Rev Sustinere. 2018;6(2):300-20.

42. Macedo KD, Acosta BS, Silva EB da, Souza NS de, Beck CLC, Silva KKD da. Metodologias ativas de aprendizagem: caminhos possíveis para inovação no ensino em saúde. Esc Anna Nery Rev Enferm. 2018;22(3):1-9.

\section{Correspondência para:}

Profa. Dra. Judith Rafaelle Oliveira Pinho e-mail: judrafa@gmail.com

Rua Barão de Itapari, 155 Centro 65020-070, São Luís/MA 